\title{
The impact of aerosols on polarized sky radiance: model development, validation, and applications
}

\author{
C. Emde ${ }^{1, *}$, R. Buras ${ }^{1, *}$, B. Mayer ${ }^{1, *}$, and M. Blumthaler ${ }^{2}$ \\ ${ }^{1}$ Institut für Physik der Atmosphäre, Deutsches Zentrum für Luft- und Raumfahrt, Oberpfaffenhofen, Germany \\ ${ }^{2}$ Sektion für Biomedizinische Physik, Medizinische Universität Innsbruck, Innsbruck, Austria \\ * now at: Meteorologisches Institut, Ludwig-Maximilians-Universität, München, Germany
}

Received: 6 May 2009 - Published in Atmos. Chem. Phys. Discuss.: 28 August 2009

Revised: 19 November 2009 - Accepted: 5 January 2010 - Published: 18 January 2010

\begin{abstract}
Although solar radiation initially is unpolarized when entering the Earth's atmosphere, it is polarized by scattering processes with molecules, water droplets, ice crystals, and aerosols. Hence, measurements of the polarization state of radiation can be used to improve remote sensing of aerosols and clouds. The analysis of polarized radiance measurements requires an accurate radiative transfer model. To this end, a new efficient and flexible threedimensional Monte Carlo code to compute polarized radiances has been developed and implemented into MYSTIC (Monte Carlo code for the phYSically correct Tracing of photons In Cloudy atmospheres). The code has been extensively validated against published benchmark results. The polarized downwelling radiation field is calculated for various aerosol types showing the high sensitivity of polarized ultraviolet radiances to the particle microphysics. Model simulations are compared to ground based measurements and found to be qualitatively in good agreement. Quantitative differences can be attributed to the assumed aerosol models based on the OPAC aerosol database, which does not include exactly the types of aerosols that have been observed. This comparison to the measurements shows that there is a high potential to retrieve information about the aerosol type from polarized radiance measurements.
\end{abstract}

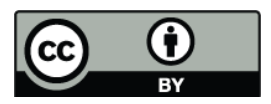

Correspondence to: C. Emde (claudia.emde@dlr.de)

\section{Introduction}

The effect of aerosols on the Earth's climate is considered as one of the largest uncertainties in our understanding of climate change (IPCC, 2007). Aerosols can affect the Earth's climate directly by scattering and absorbing solar and terrestrial radiation, or indirectly by altering cloud properties. Precise information is needed on aerosol microphysical properties such as size distribution, particle shape, refractive index, and chemical composition and on the resulting optical properties (optical thickness, single scattering albedo, and the scattering matrix) in order to quantify the aerosol impact on climate. Since there are many different aerosol types and substantial spatial and temporal variations, the retrieval of aerosol optical properties is difficult even with multiple constraints as provided by multispectral radiance measurements. Polarization measurements are highly sensitive to particle microphysics (Hansen and Travis, 1974; Boesche et al., 2006) and can help to resolve this problem because they provide additional uncorrelated constraints for the retrieval. Retrieval methods that use polarization information are described in Vermeulen and Devaux (2000); Chowdhary et al. (2004); Li et al. (2006).

Several ground based polarized radiance measurement methods exist. All-sky imaging systems have been developed to measure the polarized radiance distribution, such measurement systems are described in for instance Liu and Voss (1997); Kreuter et al. (2009) and references therein. The Research Scanning Polarimeter (RPS) (Cairns et al., 1999, 2003) has been used for ground-based as well as air-borne aerosol measurements. A scanning radiometer has been applied to measure polarized UV radiances (Blumthaler et al.,

Published by Copernicus Publications on behalf of the European Geosciences Union. 
2008) in the solar principal plane and in the almucantar plane from the ground. This radiometer can measure the angular radiance distribution including the region about the forward scattering peak accurately. Therefore the measurements have a high potential to measure not only the aerosol optical thickness but also the type of aerosol. Recently, a commercially available ground-based polarimeter, CE318-DP, has been developed by the CIMEL Electronic (Paris, France). Li et al. (2009) performed a preliminary analysis of the measurements and found that adding polarization can reduce retrieval errors in the fine mode of the size distribution, the real part of the refractive index and the particle shape parameters.

Polarized solar radiance is also measured from space. The POLarization and Directionality of Earth Reflectances (POLDER) instrument (Deschamps et al., 1994) which operated from 1996 to 1997 provided the first global measurements of the spectral, directional, and polarization characteristics of solar radiation reflected by the Earth-atmosphere system. The NASA Glory mission (Mishchenko et al., 2007) which is scheduled for launch in October 2010 will carry an Aerosol Polarimeter Sensor (APS) to determine aerosol and cloud properties. The APS sensor will provide high precision measurements of polarized reflectances in 9 narrow spectral bands between 412 and $2250 \mathrm{~nm}$ and in 240 viewing directions.

The detailed analysis of ground based as well as space borne measurements requires an accurate radiative transfer model. Most vector radiative transfer models are based on explicit methods like the discrete ordinate methods (Coulson et al., 1960; de Haan et al., 1987; Evans and Stephens, 1991; Schulz et al., 1999; Emde et al., 2004) which rely on a Legendre decomposition of the phase matrices. These methods can not handle the scattering phase matrices of e.g. large aerosol particles because the number of required Legendre terms becomes very large in the ultra-violet and visible wavelength region, resulting in extremely large differential equation systems. The number of Legendre terms can be reduced to a reasonable amount with the help of the often used Delta-M method (Wiscombe, 1977). However, this approximation is not accurate, especially in the forward scattering region. Discrete ordinate methods are also quite inefficient when they are applied to a three-dimensional atmosphere including detailed cloud structures.

Monte Carlo methods can consider accurate phase matrices and three-dimensional atmospheres without numerical problems. So far there are only a few Monte Carlo codes that consider polarization. Collins et al. (1972) developed an efficient code in the 1970s, but at this time computing power allowed to use the code only for simple atmospheres with only a few layers. The model by Davis et al. (2005) has been developed for the millimeter and submillimeter wavelength region, i.e. it can not be applied for solar radiative transfer. A model that handles 3-D atmospheres and polarization in the UV/visible wavelength region has been developed recently by Cornet et al. (2010).
This study describes the newly developed Monte Carlo code, it shows a validation and several simulations of polarized sky radiances. Simulations are shown for pure Rayleigh atmospheres, for different aerosol types, and for a water cloud. The error caused by applying the Delta-M method of the phase matrix is investigated. Model simulations are compared to ground based polarized radiance measurements that were taken in Izana, Tenerife, in June 2005.

\section{Methodology}

The radiative transfer model MYSTIC (Monte Carlo code for the phYSically correct Tracing of photons In Cloudy atmospheres; Mayer, 2009) is used for this study. MYSTIC is operated as one of several radiative transfer solvers of the libRadtran radiative transfer package (Mayer and Kylling, 2005). Originally, MYSTIC was developed as a forward tracing method for the calculation of irradiances and radiances in 3-D plane-parallel atmospheres. Recently, the model has been extended to include spherical geometry and a backward tracing mode (Emde and Mayer, 2007). For this study, the model has been further extended to include polarized radiation due to scattering by randomly oriented particles, i.e. clouds, aerosols, and molecules. For general questions about the Monte Carlo technique and in particular about libRadtran and MYSTIC the reader is referred to the literature (Marchuk et al., 1980; Collins et al., 1972; Mayer, 2009; Marshak and Davis, 2005; Cahalan et al., 2005).

\subsection{Polarized Monte Carlo model}

Additions to a scalar Monte Carlo model that are required to implement polarization by Rayleigh, aerosol, and cloud scattering are described in the following.

To implement polarization, one possibility is to assign a random polarization state to each traced photon entering the atmosphere. Another more efficient possibility is to interpret each traced photon as a photon package and to assign a weight vector corresponding to the Stokes parameters to each package. The following example demonstrates why the weight vector method is more efficient: Circular polarization is not influenced by the atmosphere, so the average of the circular component of the polarization state is zero before the photons enter the atmosphere and it will still be negligible when the photons are measured at the surface. The contributions by left and right circular polarized photons to the total circular polarisation cancel each other. In the weight vector method all photon packages contribute to the result, therefore we have implemented this method.

The Stokes parameters are defined as time averages of linear combinations of the electromagnetic field vector (Chandrasekhar, 1950; Hansen and Travis, 1974; Mishchenko et al., 2002):

$$
I=\left\langle\boldsymbol{E}_{1} \boldsymbol{E}_{1}^{*}+\boldsymbol{E}_{\mathrm{r}} \boldsymbol{E}_{\mathrm{r}}^{*}\right\rangle,
$$


$Q=\left\langle\boldsymbol{E}_{1} \boldsymbol{E}_{1}^{*}-\boldsymbol{E}_{\mathrm{r}} \boldsymbol{E}_{\mathrm{r}}^{*}\right\rangle$,

$U=\left\langle\boldsymbol{E}_{1} \boldsymbol{E}_{\mathrm{r}}^{*}+\boldsymbol{E}_{\mathrm{r}} \boldsymbol{E}_{1}^{*}\right\rangle$,

$V=i\left\langle\boldsymbol{E}_{1} \boldsymbol{E}_{\mathrm{r}}^{*}-\boldsymbol{E}_{\mathrm{r}} \boldsymbol{E}_{1}^{*}\right\rangle$.

Here, $\boldsymbol{E}_{1}$ and $\boldsymbol{E}_{\mathrm{r}}$ are the components of the electric field vector parallel and perpendicular to the reference plane respectively. The model coordinate system is defined by the vertical (z-axis), the Southern direction (x-axis) and the Eastern direction (y-axis). The Stokes vector is defined in the reference frame defined by the $\mathrm{z}$-axis and the propagation direction of the radiation. Since the Stokes parameters are real-valued and have the dimension of intensity, they can be measured directly with suitable instruments. The Stokes parameters define a complete set of quantities needed to characterize a plane electromagnetic wave as they carry information of the complex amplitudes and the phase difference.

The initial Stokes weight vector for solar radiation is $\boldsymbol{I}_{w}^{0}=$ $(1,0,0,0)$ corresponding to the unpolarized extraterrestrial solar radiation. The initial direction of the photon is defined by the solar zenith angle and the solar azimuth angle.

For randomly oriented particles absorption does not depend on the polarization state of the radiation. In MYSTIC absorption is considered by reducing the photon weight $w_{a}$ according to Lambert-Beer's Law :

$w_{a}=\exp \left(-\int \beta_{\mathrm{abs}} \mathrm{d} s\right)$.

Here $\mathrm{d} s$ is a path element of the photon path and $\beta_{\mathrm{abs}}$ is the total absorption coefficient including molecules, aerosols, and water and ice clouds.

The free path of a photon until a scattering interaction takes place is sampled according to the probability density function (PDF)

$$
P_{s}=\exp \left(-\int_{0}^{s} \beta_{\text {sca }} \mathrm{d} s^{\prime}\right) \text {. }
$$

Here, $\beta_{\text {sca }}=\sum_{i=1}^{N} \beta_{\text {sca, i }}$ is the total scattering coefficient of $N$ interacting particles and molecules. This formula is valid for randomly oriented particles (i.e. with diagonal extinction matrix).

We use a random number $r \in[0,1]$ to decide which interaction takes place. If there are $N$ types of particles and molecules at the place of scattering, the photon interacts with the $j$ th type if the random number fulfills the following condition:

$\frac{\sum_{i=1}^{j-1} \beta_{\mathrm{sca}, i}}{\beta_{\mathrm{sca}}}<r \leq \frac{\sum_{i=1}^{j} \beta_{\mathrm{sca}, i}}{\beta_{\mathrm{sca}}}$.

The next step is to sample the new photon direction. For scalar radiative transfer and randomly oriented particles the scattering direction depends only on the scattering zenith angle, not on the azimuth. Then the scattering phase function is used as the PDF to sample the new direction.

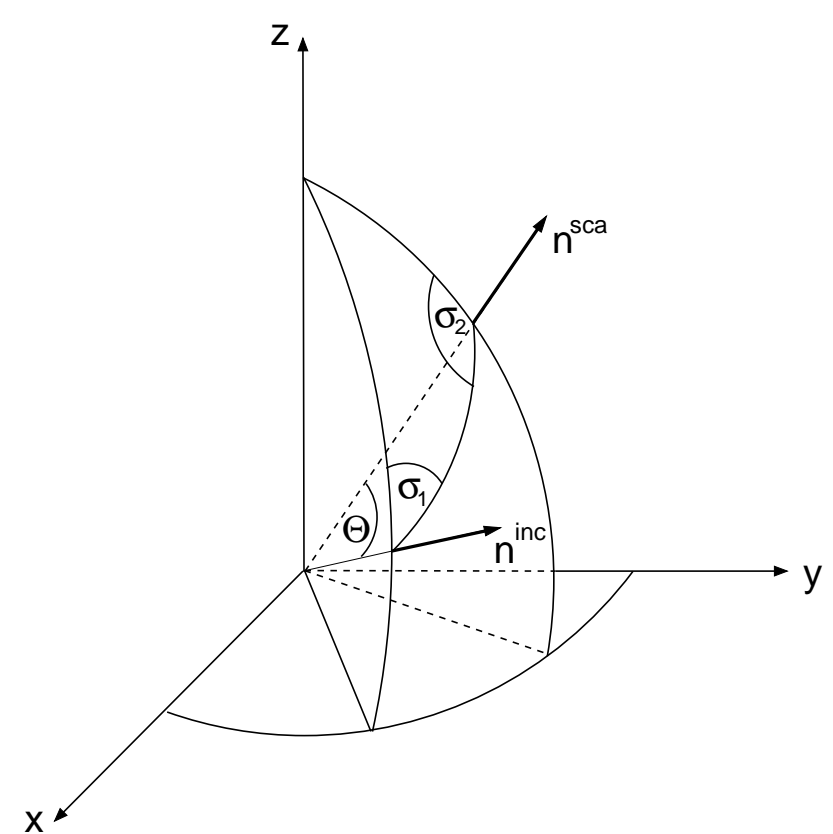

Fig. 1. Incoming $\boldsymbol{n}^{\text {inc }}$ and scattered $\boldsymbol{n}^{\text {sca }}$ directions in the model coordinate system $(\mathrm{x}, \mathrm{y}, \mathrm{z}), \sigma_{1}$ and $\sigma_{2}$ are the rotation angles to transform the Stokes vector from the reference frame to the scattering frame and vice versa and $\Theta$ is the scattering angle. The scattering plane is defined by the vectors $\boldsymbol{n}^{\text {inc }}$ and $\boldsymbol{n}^{\text {sca }}$. Figure adapted from Mishchenko et al. (2002).

For polarized radiative transfer the scattering interaction is described by the scattering phase matrix. For convenience the scattering phase matrix is defined in the scattering frame, i.e. the coordinate system defined by the plane of the incoming $\boldsymbol{n}^{\text {inc }}$ and the scattered $\boldsymbol{n}^{\text {sca }}$ photon directions and a vector orthogonal to this plane (see Fig. 1), where the phase matrix depends only on the scattering angle $\Theta$. For randomly oriented particles, six matrix elements are required (van de Hulst, 1981):

$\mathbf{P}(\Theta)=\left[\begin{array}{cccc}\mathbf{P}_{11}(\Theta) & \mathbf{P}_{12}(\Theta) & 0 & 0 \\ \mathbf{P}_{12}(\Theta) & \mathbf{P}_{22}(\Theta) & 0 & 0 \\ 0 & 0 & \mathbf{P}_{33}(\Theta) & \mathbf{P}_{34}(\Theta) \\ 0 & 0 & -\mathbf{P}_{34}(\Theta) & \mathbf{P}_{44}(\Theta)\end{array}\right]$,

while for spherical particles only four elements are required because $\mathbf{P}_{11}=\mathbf{P}_{22}$ and $\mathbf{P}_{33}=\mathbf{P}_{44}$. The Stokes vector is defined in the reference coordinate system in the plane given by the propagation direction of the radiation and the z-axis. Hence, to apply the phase matrix to the Stokes vector one has to rotate the Stokes vector into the scattering frame. After multiplication with the phase matrix it is rotated back to the reference coordinate system:

$\boldsymbol{I}^{\mathrm{sca}}=\boldsymbol{L}\left(\sigma_{2}\right) \mathbf{P} \boldsymbol{L}\left(\sigma_{1}\right) \boldsymbol{I}^{\mathrm{inc}}=\boldsymbol{Z} \boldsymbol{I}^{\mathrm{inc}}$,

where $\boldsymbol{I}^{\text {inc }}$ and $\boldsymbol{I}^{\text {sca }}$ are the incoming and scattered Stokes vectors respectively, $\sigma_{1}$ and $\sigma_{2}$ are the rotation angles for 
the Stokes vector, $\boldsymbol{Z}$ is the scattering matrix and $\boldsymbol{L}(\alpha)$ is the Stokes rotation matrix:

$\boldsymbol{L}(\alpha)=\left[\begin{array}{cccc}1 & 0 & 0 & 0 \\ 0 & \cos (2 \alpha) & -\sin (2 \alpha) & 0 \\ 0 & \sin (2 \alpha) & \cos (2 \alpha) & 0 \\ 0 & 0 & 0 & 1\end{array}\right]$.

The rotation angles $\sigma_{1}$ and $\sigma_{2}$ are calculated from the incoming $\boldsymbol{n}^{\text {inc }}\left(\theta^{\text {inc }}, \phi^{\text {inc }}\right)$ and the scattered $\boldsymbol{n}^{\text {sca }}\left(\theta^{\text {sca }}, \phi^{\text {sca }}\right)$ directions as follows:

$\sigma_{1}=\arccos \left(\left(\boldsymbol{n}^{\mathrm{inc}} \times \boldsymbol{n}^{\mathrm{z}}\right) \cdot\left(\boldsymbol{n}^{\mathrm{inc}} \times \boldsymbol{n}^{\mathrm{sca}}\right)\right)$,

$\sigma_{2}=\arccos \left(\left(\boldsymbol{n}^{\mathrm{sca}} \times \boldsymbol{n}^{\mathrm{z}}\right) \cdot\left(\boldsymbol{n}^{\mathrm{sca}} \times \boldsymbol{n}^{\mathrm{inc}}\right)\right)$.

Here, $\boldsymbol{n}^{\mathrm{z}}$ is the unit vector in $\mathrm{z}$-direction $(0,0,1)$.

The scattering matrix $\mathbf{Z}$ is the probability density matrix for sampling the new photon direction. Eq. (9) shows that $\mathbf{Z}$ depends on four angles $\left(\theta^{\text {inc }}, \phi^{\text {inc }}, \theta^{\text {sca }}, \phi^{\text {sca }}\right)$, not only on the scattering angle $\Theta$ as does the phase function in the scalar model. Nevertheless we may use $\mathbf{P}_{11}$ as PDF for the scattering angle and a random angle between 0 and $2 \pi$ for the azimuth direction to sample the scattered direction. The scattered Stokes weight vector is then given by

$\boldsymbol{I}_{w}^{\mathrm{sca}}=\mathbf{P}_{11}^{-1} \boldsymbol{L}\left(\sigma_{2}\right) \mathbf{P} \boldsymbol{L}\left(\sigma_{1}\right) \boldsymbol{I}_{w}^{\mathrm{inc}}=\mathbf{P}_{11}^{-1} \mathbf{Z} \boldsymbol{I}_{w}^{\mathrm{inc}}$,

where the weight $\mathbf{P}_{11}^{-1}$ corrects for using $\mathbf{P}_{11}$ as PDF to sample the scattered direction. This method is also known as importance sampling (Marchuk et al., 1980). A more detailed description of the application of importance sampling to sample the scattering direction in polarized radiative transfer can be found in Collins et al. (1972).

After $n$ scattering events the Stokes weight vector is

$\boldsymbol{I}_{w}^{n}=\left(\prod_{i=n}^{1}\left(\mathbf{P}_{11}^{-1}\right)_{i} \mathbf{Z}_{i}\right) \boldsymbol{I}_{w}^{0}$,

where $i$ denotes the number of the scattering event. We trace the photon through the atmosphere, either from the sun to the sensor (forward tracing) or from the sensor to the sun (backward tracing) and calculate the product of all scattering matrices. Here it is important that the matrix multiplications are in the correct order as in Eq. (14). The total scattering matrix is then multiplied with the initial Stokes weight vector to get the final Stokes weight vector.

In order to reduce the noise of radiance calculations the local estimate method has also been implemented. A detailed mathematical derivation for scalar radiative transfer is given in Marshak and Davis (2005). The method can easily be adapted to polarized radiative transfer.

\subsection{Rayleigh scattering}

Molecular scattering is described by the analytical phase matrix $\mathbf{P}_{\mathrm{R}}$ (Hansen and Travis, 1974)

$$
\begin{aligned}
& \mathbf{P}_{\mathrm{R}}(\Theta)= \\
& \Delta\left[\begin{array}{cccc}
\frac{3}{4}\left(1+\cos ^{2} \Theta\right) & -\frac{3}{4} \sin ^{2} \Theta & 0 & 0 \\
-\frac{3}{4} \sin ^{2} \Theta & \frac{3}{4}\left(1+\cos ^{2} \Theta\right) & 0 & 0 \\
0 & 0 & \frac{3}{2} \cos \Theta & 0 \\
0 & 0 & 0 & \Delta^{\prime} \frac{3}{2} \cos \Theta
\end{array}\right] \\
& +(1-\Delta)\left[\begin{array}{llll}
1 & 0 & 0 & 0 \\
0 & 0 & 0 & 0 \\
0 & 0 & 0 & 0 \\
0 & 0 & 0 & 1
\end{array}\right],
\end{aligned}
$$

where

$\Delta=\frac{1-\delta}{1+\delta / 2}, \quad \Delta^{\prime}=\frac{1-2 \delta}{1-\delta}$,

and $\delta$ is the depolarization factor that accounts for the anisotropy of the molecules. The scattering and the absorption coefficients can also be derived analytically.

\subsection{Water cloud scattering}

The optical properties of water cloud droplets can be calculated using Mie theory (Mie, 1908). For this study we have applied the well known and well validated Mie code by Wiscombe (1980). We have assumed that the particle sizes are gamma-distributed:

$n(r)=C r^{\alpha} \exp \left(-\frac{(\alpha+3) r}{r_{\mathrm{eff}}}\right)$

Here $\alpha$ was set 7 and the effective radius $r_{\text {eff }}$ was $10 \mu \mathrm{m}$. The effective variance is $v_{\text {eff }}=\frac{1}{\alpha+3}=0.1$ which is a reasonable value for a cloud. The constant $C$ has been determined by normalisation. The size distribution has been cut off at a minimum radius of $0.2 \mu \mathrm{m}$ and a maximum radius of $80 \mu \mathrm{m}$. The refractive index of water has been taken from Warren (1984), it is $1.358-2.52^{-9} i$ at a wavelength of $350 \mathrm{~nm}$.

\subsection{Aerosol scattering}

The optical properties of aerosols were also calculated using Mie theory (Mie, 1908). The refractive index of the aerosol particles and the size distributions were taken from the OPAC aerosol database (Hess et al., 1998) for various aerosol types. OPAC also provides typical aerosol type mixtures, for instance for continental polluted or desert conditions.

Figure 2 shows the phase matrices of various OPAC aerosol types, of liquid cloud droplets with an effective radius of $10 \mu \mathrm{m}$ and, for comparison, the Rayleigh scattering phase matrix. OPAC provides parameters for the log-normal distribution

$n(r)=\frac{C}{r} \exp \left[-\frac{1}{2}\left(\frac{\ln r-\ln r_{\mathrm{mod}}}{\ln \sigma}\right)^{2}\right]$, 
Table 1. Parameters from the OPAC database (Hess et al., 1998) for $350 \mathrm{~nm}$ that were used for Mie calculations to obtain the aerosol scattering phase matrices shown in Fig. 2.

\begin{tabular}{lccccccc}
\hline Component & $\begin{array}{c}\text { relative humidity } \\
(\%)\end{array}$ & $\begin{array}{c}r_{\text {mod }} \\
(\mu \mathrm{m})\end{array}$ & $\begin{array}{c}r_{\min } \\
(\mu \mathrm{m})\end{array}$ & $\begin{array}{c}r_{\max \max } \\
(\mu \mathrm{m})\end{array}$ & $\sigma$ & $\begin{array}{c}\rho \\
\left(\mathrm{g} \mathrm{cm}^{-3}\right)\end{array}$ & refractive index \\
\hline Water soluble & 95 & 0.0399 & 0.0074 & 42.8 & 2.24 & 1.12 & $1.37-7.50^{-4} i$ \\
Sea salt (accumulated mode) & 95 & 0.605 & 0.0108 & 58.5 & 2.03 & 1.05 & $1.35-1.96^{-8} i$ \\
Soot & 0 & 0.0118 & 0.005 & 20.0 & 2.00 & 1.00 & $1.75-4.65^{-1} i$ \\
\hline
\end{tabular}

where $r_{\text {mod }}$ is the mode radius, $\sigma$ measures the width of the distribution, and the constant $C$ is determined by normalisation. Table 1 shows the parameters that were used to compute the phase matrices shown in Fig. 2. OPAC parameters for altogether 10 aerosol types and various relative humidities have been used to produce a dataset of aerosol optical properties including the complete phase matrices so that the MYTSIC model allows to define arbitrary mixtures of the basic aerosol types and uses automatically aerosol properties for the relative humidity corresponding to the background atmosphere.

Figure 2 shows that the forward scattering peak increases with particle size. Rayleigh scattering is described by the electromagnetic field of an oscillating dipole with isotropic polarizability (van de Hulst, 1981), therefore the phase matrix element $\mathbf{P}_{12}$ for Rayleigh scattering has the minimum at $90^{\circ}$. This means that the linear polarization has its maximum at a scattering angle of $90^{\circ}$. For soot, this minimum is still visible but for other aerosols and for the cloud droplets the angular dependence of $\boldsymbol{P}_{12}$ is completely different which means that we may expect completely different polarization characteristics.

\subsection{Lambertian surface}

For polarized radiation, MYSTIC can currently handle Lambertian surfaces and reflectance matrices. For this study simulations were performed with Lambertian surfaces only. According to Lambert's law the diffusely reflected light is isotropic and is unpolarized, independently of the state of polarization of the incident radiation and the angle of illumination (Chandrasekhar, 1950).

\subsection{Model validation}

For validation the newly developed polarization module of the MYSTIC Monte Carlo model has been applied to different atmospheric conditions and compared to benchmark results.

For Rayleigh scattering we compared to results tabulated by Coulson et al. (1960). These results are for a planeparallel Rayleigh atmosphere with one layer. They have been obtained following a solution method proposed by Chandrasekhar (1950) that is entirely different from the Monte
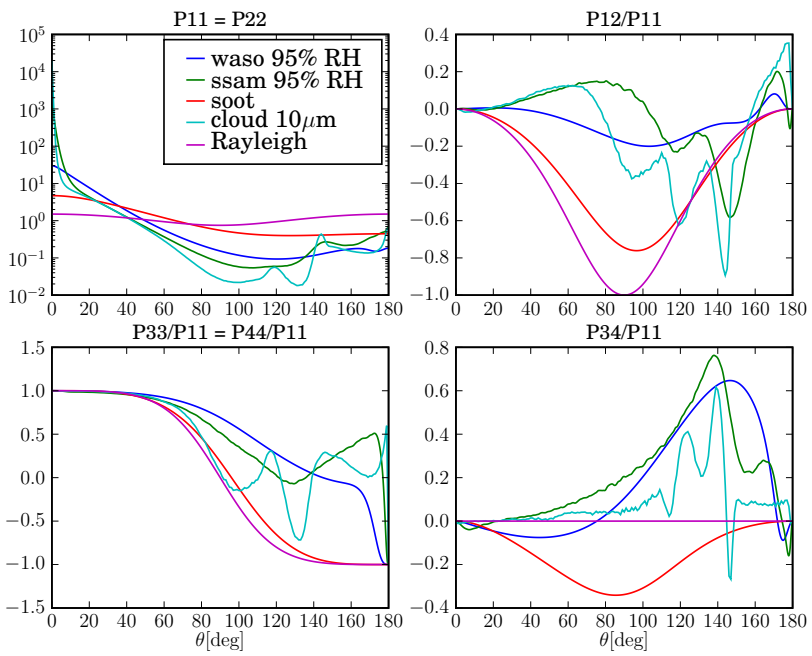

Fig. 2. Phase matrix elements for OPAC aerosol types "water soluble" (WASO), "sea salt accumulated mode" (SSAM), and soot, for a liquid water cloud with a droplet effective radius of $10 \mu \mathrm{m}$, and for Rayleigh scattering. The wavelength is $350 \mathrm{~nm}$.

Carlo method. A typical result of the comparison is shown in Fig. 3. There is a perfect agreement between MYSTIC and the results by Coulson. The relative difference is smaller than $0.5 \%$ and well within the Monte Carlo noise. To achieve this accuracy $10^{7}$ photons were required. The error bars in the figure correspond to two standard deviations.

In order to check whether the model works correctly for non-spherical randomly oriented aerosol particles we compared to benchmark results tabulated by Wauben and Hovenier (1992) who calculated multiple-scattered polarized radiances for a homogeneous plane-parallel layer using two independent methods: (1) a doubling and adding method and (2) the so-called $F_{N}$ method. As an example we show the comparison for aerosol model 1 of Kuik et al. (1992), which consists of randomly-oriented prolate spheroid with aspect ratio (the ratio of major and minor axis) 4.0, size parameter $(\pi$ times the major axis divided by the wavelength) 10.079368 and refractive index $1.55-0.01 i$. Figure 4 shows results of reflected and transmitted radiances. The extraterrestrial irradiance is set to $\pi$ and the cosine of the solar zenith angle is 0.6. The surface albedo is 0 , i.e. perfectly absorbing. The 

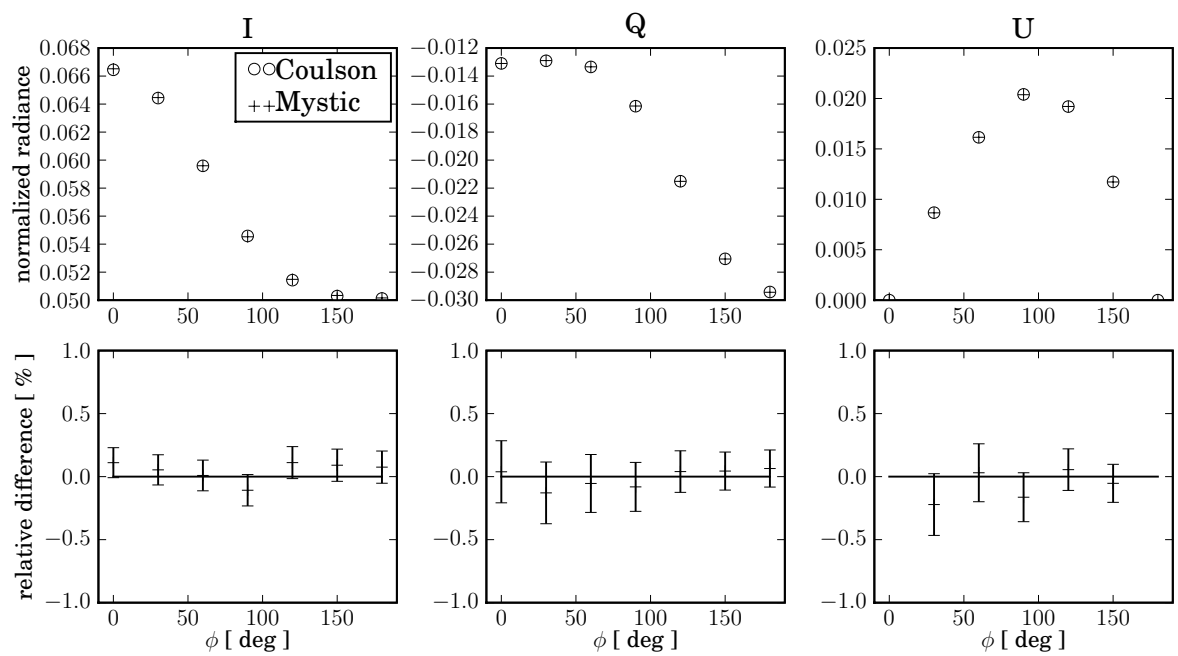

Fig. 3. Comparison between MYSTIC and tabulated values by Coulson et al. (1960) for a one layer Rayleigh scattering atmosphere. Shown are downwelling radiances at the surface that are normalized to the extraterrestrial solar radiation. The circles show the results by Coulson and the crosses are the MYSTIC results. The x-axis is the azimuth angle of the viewing direction. The cosine of the solar zenith angle is 0.92 , the optical thickness 0.25 , the surface albedo is 0.25 , and the cosine of the viewing zenith angle is 0.4 . The error bars correspond to two standard deviations.

relative azimuth angles between radiance direction and incoming direction are $0^{\circ}$ and $90^{\circ}$ respectively. Figure 4 shows that MYSTIC agrees very well with the benchmark results. Relative differences are less than $0.5 \%$ for the Stokes components $I, Q$ and $U$. For the circular component $V$ the relative differences are larger, up to 5\%, mainly due to the very small absolute values of $V$ (about 2 orders of magnitude smaller than $Q$ and $U$ ). All differences can be explained by the inherent statistical noise of the Monte Carlo method and could be decreased by running more photons. The results shown here were obtained with $10^{7}$ photons.

Benchmark results for an inhomogeneous atmosphere above a Lambertian surface have been provided by de Haan et al. (1987). The inhomogeneous atmosphere has an optical thickness of 0.6 and consists of two layers with the following specifications: The upper layer consists of molecules only and has an optical thickness of 0.1. The lower layer consists of a mixture of molecules and haze droplets (water-haze $\mathrm{L}$ as defined by de Rooij and van der Stap, 1984), specified by a molecular optical thickness of 0.1 and an aerosol optical thickness of 0.4. The surface is Lambertian with an albedo of 0.1 . The depolarization factor was set to 0.0279 , corresponding to air. It is furthermore assumed that the molecules do not absorb. Table 2 shows results by de Haan et al. (1987) and corresponding MYSTIC results that were obtained using $10^{7}$ photons. The errors correspond to $2 \sigma$. There is an excellent agreement between MYSTIC and de Haan et al. (1987), all deviations are within the Monte Carlo noise. The comparison has also been made for all other inhomogeneous test cases by de Haan et al. (1987) and the agreement was always excellent.
The very good agreement between MYSTIC and benchmark results for Rayleigh and aerosol scattering gives confidence in our approach to compute polarized radiances.

\subsection{Program efficiency}

The model is implemented in $\mathrm{C}$ and its computation time (CPU time) has been tested on an Intel Pentium processor with $2.80 \mathrm{GHz}$. The computation time depends mostly on the optical thickness. Table 3 shows CPU times at $500 \mathrm{~nm}$ wavelength where the Rayleigh optical thickness is approximately 0.15 . For the aerosol calculation, water soluble aerosol (OPAC-WASO) with an optical thickness of 0.2 was included. It can be seen that for MYSTIC the time difference between scalar and vector calculations is relatively small.

\section{Model simulations}

We performed simulations for different setups in order to understand the influence of the various atmospheric components on the polarization.

In the first section we show simulations of the full sky radiance field as observed from the surface. In order to be able to separate the polarization patterns of the different atmospheric components, we first did simulations for atmospheres including: (1) only molecules, (2) only water soluble aerosol as defined in OPAC, (3) only sea salt, and (4) only cloud droplets. Another realistic simulation shows the sky radiance for a molecular atmosphere including a typical continental aerosol mixture. The given optical thicknesses always refer to the optical thickness at the wavelength of interest. 
Table 2. Comparison against benchmark results by de Haan et al. (1987). The errors correspond to two standard deviations.

\begin{tabular}{l|rr|rr|rr}
\hline & & & & $\mu=0.5$ & & $\mu=1.0$ \\
& de Haan & MYSTIC & de Haan & MYSTIC & de Haan & MYSTIC \\
\hline$\phi=0^{\circ}$ & 0.532950 & $0.53312 \pm 0.00061$ & 0.208430 & $0.20845 \pm 0.00025$ & 0.093680 & $0.09371 \pm 0.00011$ \\
$\mu_{0}=0.5$ & -0.028340 & $-0.02818 \pm 0.00015$ & -0.036299 & $-0.03640 \pm 0.00007$ & -0.024156 & $-0.02422 \pm 0.00003$ \\
& 0.000000 & $0.00002 \pm 0.00012$ & 0.000000 & $-0.00001 \pm 0.00005$ & 0.000000 & $0.00001 \pm 0.00002$ \\
& 0.000000 & $0.00000 \pm 0.00001$ & 0.000000 & $0.00000 \pm 0.00001$ & 0.000000 & $0.00000 \pm 0.00000$ \\
\hline$\phi=30^{\circ}$ & 0.418140 & $0.41848 \pm 0.00053$ & 0.184970 & $0.18493 \pm 0.00024$ & 0.093680 & $0.09363 \pm 0.00011$ \\
$\mu_{0}=0.5$ & -0.000058 & $-0.00000 \pm 0.00014$ & -0.019649 & $-0.01973 \pm 0.00006$ & -0.012078 & $-0.01210 \pm 0.00002$ \\
& -0.073705 & $-0.07336 \pm 0.00016$ & -0.041401 & $-0.04147 \pm 0.00007$ & -0.020920 & $-0.02097 \pm 0.00003$ \\
& 0.000106 & $0.00010 \pm 0.00001$ & 0.000040 & $0.00005 \pm 0.00001$ & 0.000000 & $-0.00000 \pm 0.00000$ \\
\hline
\end{tabular}
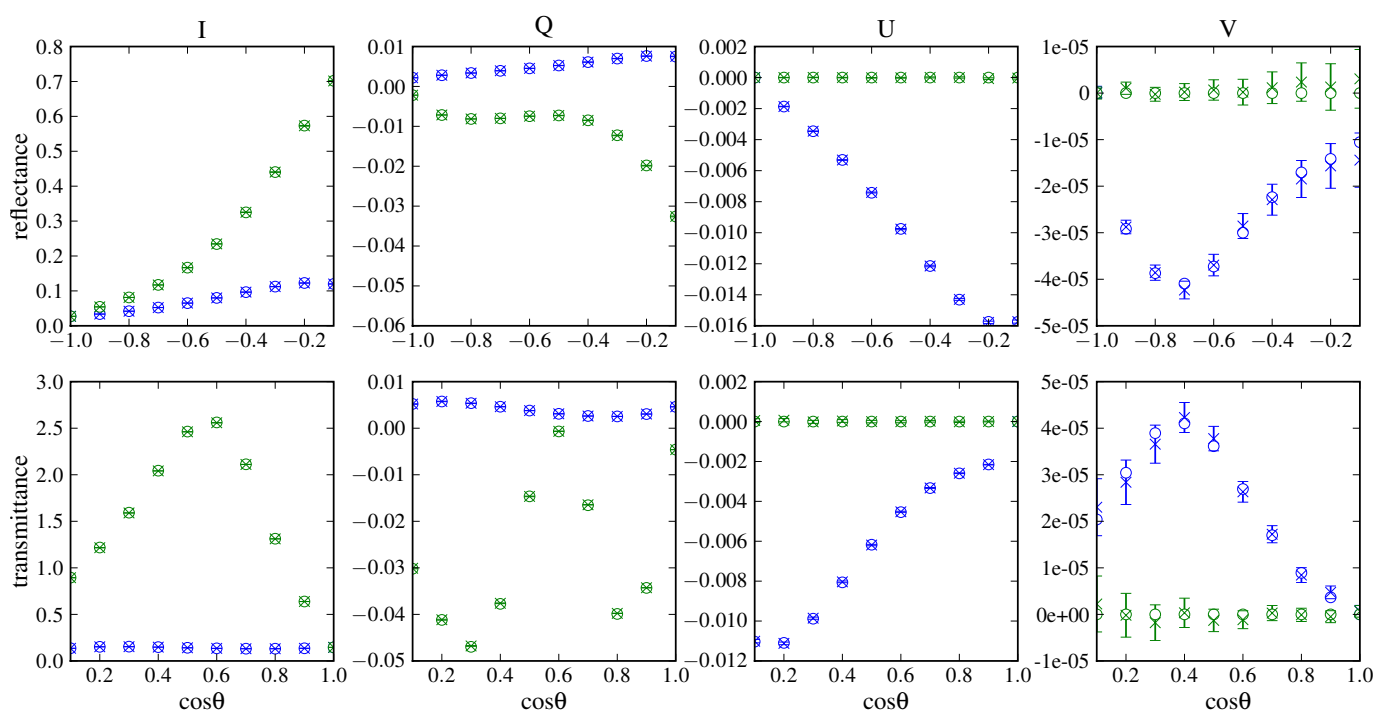

Fig. 4. Comparison between MYSTIC and tabulated values by Wauben and Hovenier (1992) for a plane-parallel aerosol layer. The upper panels show the reflectance at the top of the layer and the lower panels show the transmittance at the bottom of the layer, all as a function of viewing zenith angle $\theta$. All values are for an incoming irradiance of $\pi$ as in Wauben and Hovenier (1992). The cosine of the solar zenith angle is 0.6. The circles show the results by Wauben and Hovenier (1992) and the crosses show the MYSTIC results. Green and blue markers are for viewing azimuth angles of $0^{\circ}$ and $90^{\circ}$ respectively. The error bars correspond to two standard deviations.

In the next sections we quantitatively investigate Rayleigh scattering in the solar principal and in the almucantar plane, the dependence of the degree of polarization on the surface albedo, and the influence of aerosol scattering on the polarized radiance field.

\subsection{Polarized radiance field}

Figure 5 shows simulated Stokes components and the degree of polarisation at the surface for a wavelength of $350 \mathrm{~nm}$. The solar zenith angle is $30^{\circ}$ and the solar azimuth angle is $0^{\circ}$. The surface albedo is 0 . The simulations in this section were done in plane-parallel geometry. The values are normalized to the extraterrestrial irradiance. The degree of polarization is defined as
Table 3. Computation times for 100 calculations at $500 \mathrm{~nm}$ wavelength for one radiance direction. The accuracy corresponding to the standard deviation is also given.

\begin{tabular}{|c|c|c|}
\hline settings & Rayleigh & Rayleigh+aerosol \\
\hline \multicolumn{3}{|c|}{ vector calculation } \\
\hline $10^{4}$ photons & $2.0 \%, 22 \mathrm{~s}$ & $1.9 \%, 3 \mathrm{~m} 27 \mathrm{~s}$ \\
\hline $10^{5}$ photons & $0.6 \%, 2 \mathrm{~m} 51 \mathrm{~s}$ & $0.6 \%, 7 \mathrm{~m} 45 \mathrm{~s}$ \\
\hline \multicolumn{3}{|c|}{ scalar calculation } \\
\hline $10^{4}$ photons & $2.0 \%, 22 \mathrm{~s}$ & $1.9 \%, 3 \mathrm{~m} 19 \mathrm{~s}$ \\
\hline $10^{5}$ photons & $0.7 \%, 2 \mathrm{~m} 52 \mathrm{~s}$ & $0.6 \%, 6 \mathrm{~m} 26 \mathrm{~s}$ \\
\hline
\end{tabular}



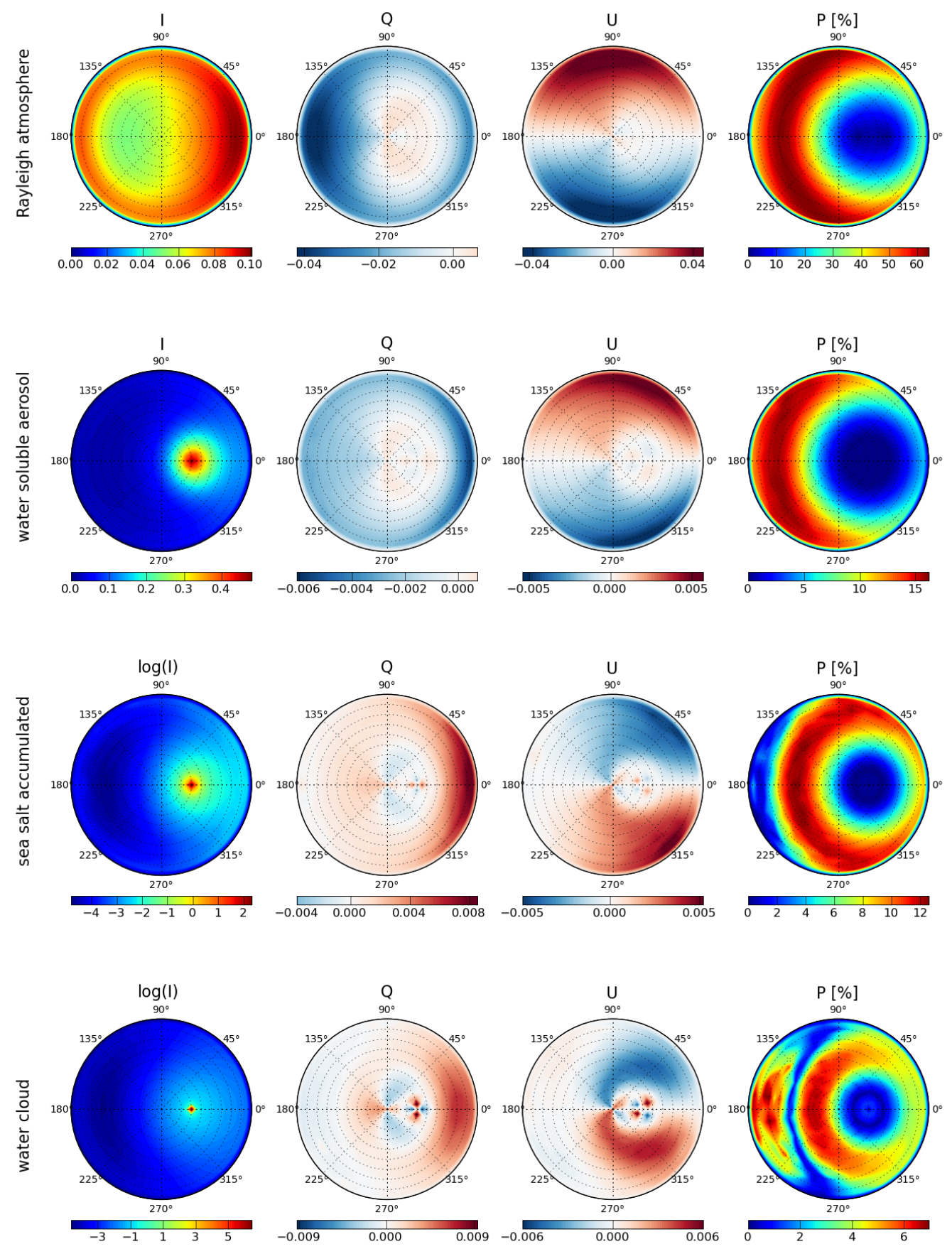

Fig. 5. Simulated Stokes components and degree of polarisation for down-welling radiation at $350 \mathrm{~nm}$ for a solar zenith angle of $30^{\circ}$. The radiance values are normalized to the extraterrestrial irradiance. The top row is for a Rayleigh scattering atmosphere, the simulations for water soluble and sea salt are for an atmosphere consisting only of aerosols, and the bottom row is for a single cloud layer with an optical thickness of 1 and an effective particle radius of $10 \mu \mathrm{m}$.

$p=\frac{\sqrt{Q^{2}+U^{2}+V^{2}}}{I}$.

The upper row shows the simulation for a molecular atmosphere (Rayleigh scattering only). The lowest intensity is obtained at viewing directions orthogonal to the sun direction. This is the expected result because the Rayleigh scat- tering phase function $\mathbf{P}_{\mathrm{R}, 11}$ has a minimum at $90^{\circ}$. Towards the horizon the intensity increases. At very shallow angles it decreases again. The degree of polarization has a maximum at directions perpendicular to the sun direction. This is expected, because the absolute value of $\mathbf{P}_{\mathrm{R}, 12}$ has a maximum at $90^{\circ}$. 

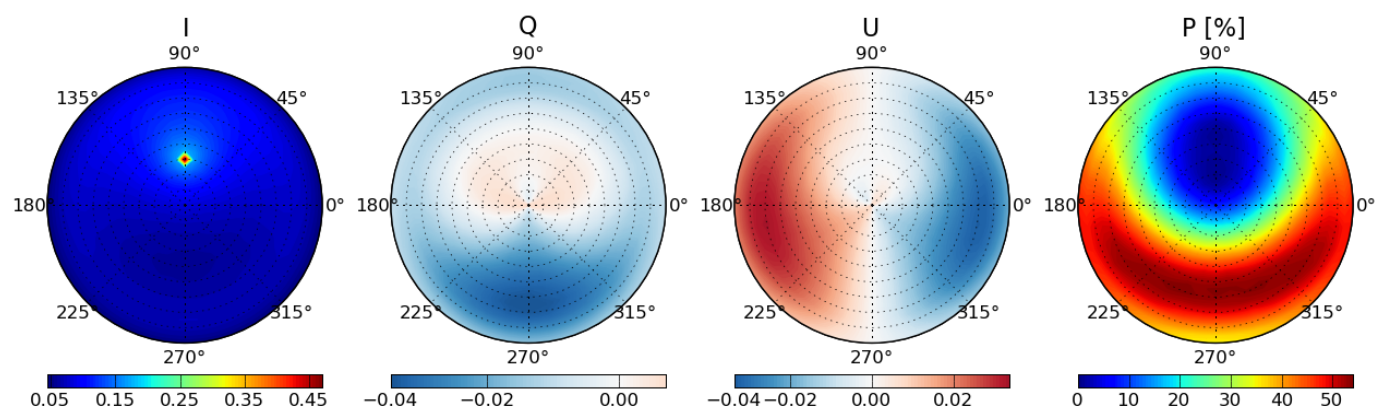

Fig. 6. Stokes components and degree of polarization at $350 \mathrm{~nm}$ for a molecular atmosphere with typical aerosol conditions ("continental average" mixture as defined in OPAC with an optical thickness of approximately 0.16 ).

In order to see how different aerosol types and clouds influence the polarized radiation field we performed simulations for hypothetical atmospheres including only aerosol ("WASO" and "SSAM" as defined in OPAC) or only cloud particles without molecules. The results are shown in rows 2 to 4 of Fig. 5. The assumed aerosol optical thickness for both aerosol simulations is 0.4 and the cloud optical thickness is 1. The effective particle radius of the water soluble aerosol is about $0.15 \mu \mathrm{m}$, of the sea salt (accumulated mode) about $1 \mu \mathrm{m}$, and of the cloud particles it is $10 \mu \mathrm{m}$. The intensity plots show clearly that the forward scattering peak becomes sharper with increasing particle size. The Stokes components $Q$ and $U$ of the calculation for water soluble aerosol show a similar pattern as for Rayleigh scattering, but their magnitudes are smaller. For larger particles (sea salt and cloud droplets) the pattern is different: large regions show opposite polarisation behaviors (sign change in $Q$ and $U$ ). This can be qualitatively explained by the ratio $\mathbf{P}_{12} / \mathbf{P}_{11}$ which is positive between approximately $0^{\circ}$ and $80^{\circ}$ for cloud particles and sea salt and negative for Rayleigh and water soluble aerosol (see Fig. 2). For the aerosol simulations the degree of polarisation has one maximum at a scattering angle larger than $90^{\circ}$ for the water soluble aerosol and at an angle less than $90^{\circ}$ for sea salt. For clouds the degree of polarisation has two maxima, one below $90^{\circ}$ scattering angle and one above.

Figure 6 shows the Stokes components and the degree of polarisation for a realistic atmosphere including molecules and the typical "continental clean" aerosol mixture as defined in OPAC with an optical thickness of approximately 0.16 . This aerosol mixture includes the basic types "water soluble", "insoluble" and "soot". The main difference between this result and the result for pure Rayleigh scattering in the intensity field is the very sharp peak in the direction of the sun due to strong forward scattering by aerosols. The pattern of the degree of polarization is very similar to Rayleigh scattering but its magnitude is decreased by approximately $20 \%$.

\subsection{Rayleigh scattering}

Ground-based measurements of sky radiances are often conducted in the solar principal plane and in the almucantar plane. Simulations of such measurements have been performed for different solar zenith angles at $350 \mathrm{~nm}$ wavelength in spherical geometry. The surface albedo is here set to 0 .

Results for the principal plane are shown in Fig. 7. The viewing angle is defined as follows: At $0^{\circ}$ the sensor points to the horizon into the direction of the sun, at $90^{\circ}$ it points to the zenith, and at $180^{\circ}$ is points to the horizon opposite to the sun. The principal plane corresponds to the horizontal line in the center of the plots in Figs. 5 and $6 . I$ is largest close to the horizon (at about $15^{\circ}$ ) independent of the solar zenith angle $\theta_{0}$. For low sun positions the variation of $I$ increases. In the principal plane, $Q$ is mostly negative with its minimum at about $\theta_{0}+90^{\circ}$. The maximum value of $Q$ is at the sun position and it is 0 if the sun is in the zenith. For other sun positions it becomes positive. In the principal plane $U$ must be exactly 0 for symmetry arguments. The small deviations from 0 are Monte Carlo noise. The maximum degree of polarization is, independent of the solar zenith angle, approximately $65 \%$. It is found at $\theta_{0}+90^{\circ}$ as expected for Rayleigh scattering.

Results for the almucantar plane are shown in Fig. 8. The almucantar plane corresponds to an azimuthal scan with a constant zenith angle corresponding to the solar zenith angle. Hence it represents a concentric circle around the zenith through the sun position in Figs. 5 and 6. At $0^{\circ}$ and $360^{\circ}$ the sensor points exactly to the sun and $180^{\circ}$ is the direction opposite to the sun. The Stokes components $Q$ and $U$ have maxima/minima at $90^{\circ}$. The degree of polarization increases with increasing solar zenith angle.

\subsection{Lambertian surface}

The influence of the surface albedo on the degree of polarisation is shown in Fig. 9. Again the principal plane is shown for a Rayleigh atmosphere at $350 \mathrm{~nm}$ wavelength. The intensity increases with increasing surface albedo whereas the degree 

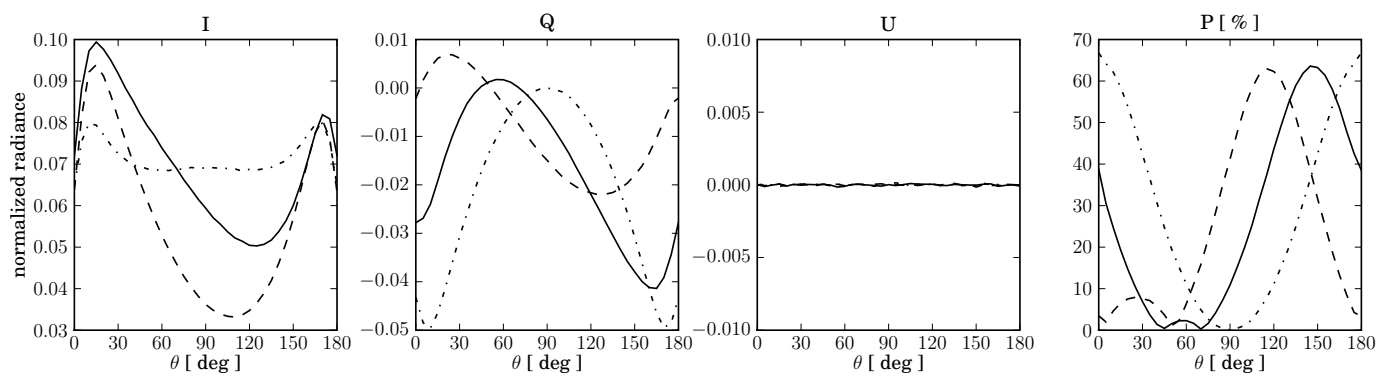

Fig. 7. Stokes components and degree of polarization at $350 \mathrm{~nm}$ for a pure Rayleigh atmosphere in the solar principal plane. Different lines correspond to different solar zenith angles: $0^{\circ}(-\cdot), 30^{\circ}(-)$, and $60^{\circ}(---)$.
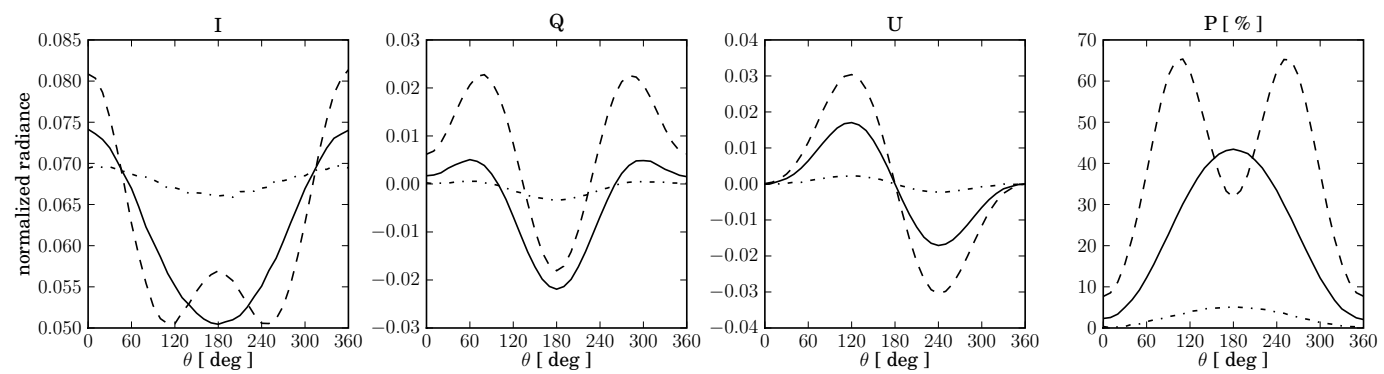

Fig. 8. Stokes components and degree of polarization at $350 \mathrm{~nm}$ for a pure Rayleigh atmosphere in the solar almucantar plane. Different lines correspond to different solar zenith angles: $10^{\circ}(-\cdots), 30^{\circ}(-)$, and $60^{\circ}(---)$.
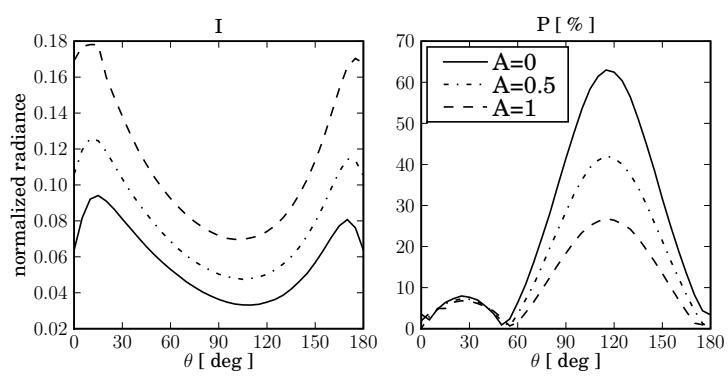

Fig. 9. Intensity and degree of polarization for a Rayleigh atmosphere assuming a Lambertian surface with different albedos. The assumed solar zenith angle is $60^{\circ}$ and the wavelength $350 \mathrm{~nm}$. The viewing directions are in the principal plane.

of polarization decreases. This is due to the assumption of a Lambertian surface that reflects isotropic unpolarized radiation.

\subsection{Aerosol simulations}

As in Sect. 3.1 aerosol simulations are performed for different types from the OPAC database. All of the following simulations include Rayleigh scattering.

Aerosol phase functions for the UV-wavelength region have sharp forward scattering peaks, especially for larger particles like sea salt. Figures 10 and 11 show simulations for the OPAC types WASO and SSAM. The figures are for aerosol optical thicknesses of 0.5 and 0.05 respectively. The left plots show the intensity in the region of the forward scattering peak. The right plots show the degree of polarization in the whole principal plane. Using MYSTIC we simulated the sky-radiance using the accurate phase matrices (solid lines). The dotted and dashed-dotted lines show MYSTIC simulations where we used Delta-M scaled optical properties. The number of Legendre terms used to approximate the phase matrix was 8 and 16, respectively.

For water soluble aerosols having effective radii well below $1 \mu \mathrm{m}$ the forward scattering peak becomes well pronounced in the radiance. The error due to Delta-M scaling in the forward scattering region is obvious for both optical thicknesses. However, the degree of polarization for water soluble aerosol can be quite accurately simulated using 8 Legendre terms in combination with the Delta-M method.

Sea salt with an effective radius of about $1 \mu \mathrm{m}$ has a sharp forward scattering peak. This peak is very large in the accurate simulation. Using the Delta-M method and 8 or 16 Legendre polynomials, the peak is almost completely removed. The correct result is several times larger than the Delta-M scaled result for both simulations. For the large optical thickness case the Delta-M method becomes inaccurate for the degree of polarization. The error is about $5 \%$. 

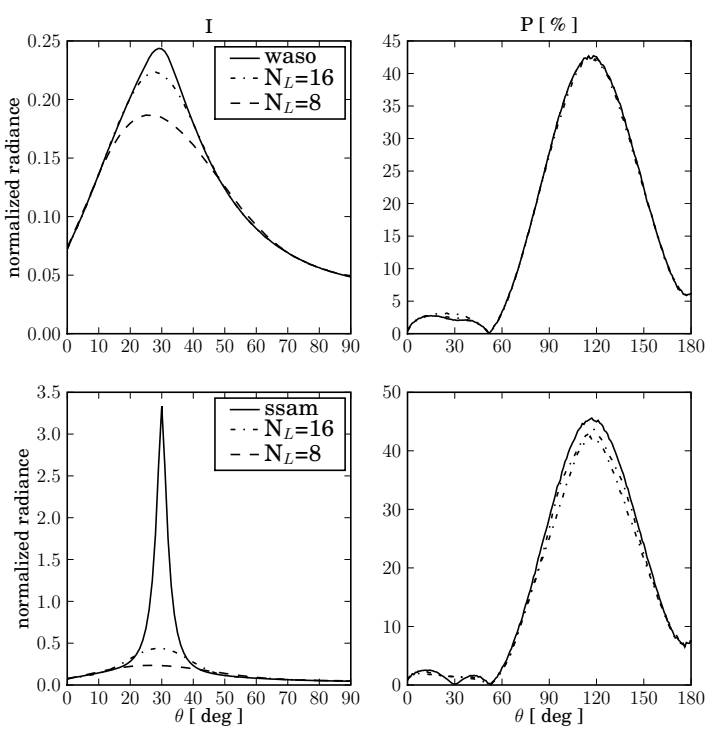

Fig. 10. Intensity and degree of polarisation in the solar principal plane for an aerosol optical thickness of 0.5 assuming that all aerosol corresponds to one aerosol type as defined in OPAC, i.e. water soluble (WASO) and sea salt accumulated mode (SSAM). The assumed solar zenith angle is $60^{\circ}$ and the wavelength is $350 \mathrm{~nm}$. The dashed and dashed-dotted lines show results using the delta-M method with 16 and 8 streams respectively.

\section{Comparison to UV radiance measurements}

We have compared our model simulations with polarized UV radiance measurements taken in Izana, Tenerife, on the 3rd and the 12th of June 2005 on a mountain top at an altitude of $2367 \mathrm{~m}$.

Besides the radiances, global irradiances were measured with an accuracy better than 5\%. The aerosol optical thickness, the ozone column, and an effective surface albedo could be derived from the irradiance measurements and were used as model input for the radiance simulations. On both days the aerosol optical thickness was found to be 0.06 . The ozone column was $305 \mathrm{DU}$ on the $3 \mathrm{rd}$ and $315 \mathrm{DU}$ on the 12 June. The surface at Izana consists of volcanic rock with little vegetation yielding a local surface albedo of less than 0.05 . By comparing the global irradiance with model simulations (for irradiance simulations we used the well tested DISORT code by Stamnes et al., 1988) we derived an effective surface albedo in order to take into account the clouds below the measurement site. This method is described in detail by Weihs et al. (2001). The effective surface albedo was 0.2 on the $3 \mathrm{rd}$ and 0.5 on the 12th of June. The solar zenith angle was calculated from the acquisition times of the measurements using the algorithm by Blanco-Muriel et al. (2001). MYSTIC can handle arbitrary mixtures of the OPAC aerosol types. For the comparison with the measurements the aerosol was modelled as a mixture of the types mineral accumulated mode $(75 \%)$ and water soluble aerosols (25\%).
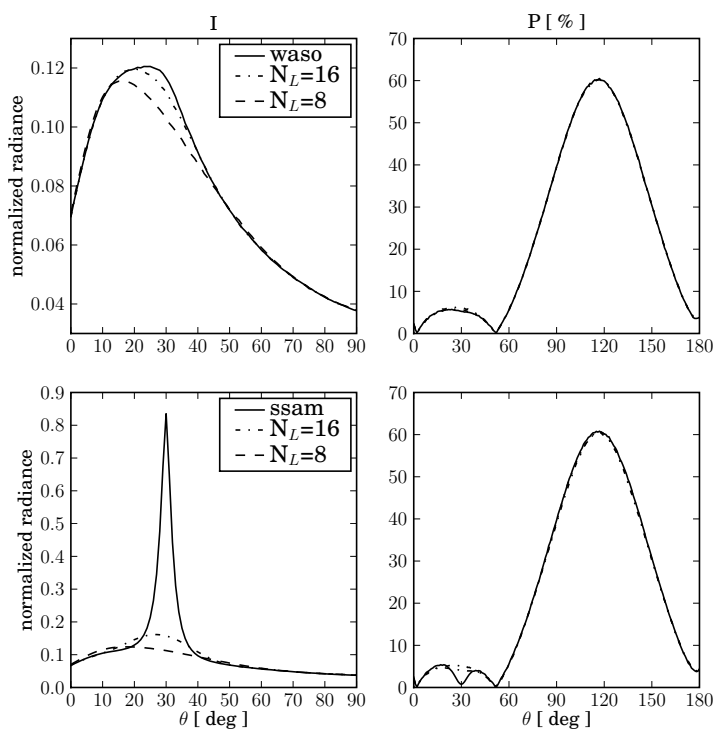

Fig. 11. Same as Fig. 10 but for an aerosol optical thickness of 0.05 .

By trying different mixtures of the OPAC aerosol types, we found that this mixture is the most appropriate to simulate the measurements.

Figures 12 and 13 show measurements and simulations of the intensity and the degree of polarization. Since there was no absolute calibration for the radiance measurements, all measured intensity values have been normalized to the model value at $120^{\circ}$ viewing angle. Unlike the radiances the degree of polarization does not depend on the calibration (see Eq. 18).

The lines in Fig. 12 show the simulations using MYSTIC and the triangles show the measurements from 3 June 2005 at wavelengths of $310 \mathrm{~nm}, 350 \mathrm{~nm}$, and $450 \mathrm{~nm}$. The measured intensity including the region of the forward scattering peak can be well reproduced by the MYSTIC model, the only difference is that the measured peak is slightly narrower than the modeled one. The forward peak is mainly caused by the mineral aerosol; the water soluble aerosol alone would yield a much smaller peak. The degree of polarization also agrees well with the model for $310 \mathrm{~nm}$ and $350 \mathrm{~nm}$. For $450 \mathrm{~nm}$ the modeled degree of polarization is lower than the measured one. A possible explanation is that the wavelength dependence of the refractive index of the aerosol which is required to compute the optical properties is not realistic. For shallow viewing angles close to $0^{\circ}$ the measured degree of polarization is larger for all wavelengths. Here the assumption of a "cloud surface" with effective surface albedo 0.2 is probably not correct. There is a clear wavelength dependence in the angular intensity distribution: The larger the wavelength the larger the intensity close to the horizon. This dependence is seen in model and measurements. The degree of polarization increases with increasing wavelength. 

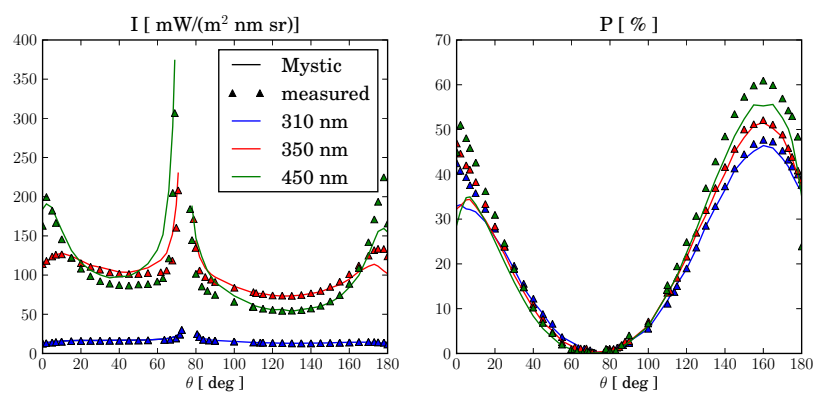

Fig. 12. Normalized intensity and degree of polarization simulated for an aerosol mixture of mineral and water soluble particles (3 June 2005, 12:00 UTC). The aerosol optical thickness was 0.06. Clouds below the measurement site are taken into account using an effective surface albedo of 0.2 .

Figure 13 shows measurements from the 12 June 2005 and model simulations. Again there is a pronounced forward scattering peak. For this day the agreement between the simulated forward scattering peak and the measured peak is not as good as for the 3 June. At other directions the modeled intensity agrees well with the measurements. Although the effective surface albedo is larger than on the 3 June the intensity is not larger because the measurement was performed earlier in the morning (larger solar zenith angle). The degree of polarization is clearly smaller than on the 3 June which can not be due to the different solar zenith angle (see Fig. 7). The explanation is simply that there were more clouds below the measurement site on the 12 June compared to the 3 June, increasing the effective surface albedo to 0.5 . This decreases the degree of polarization as shown in Fig. 9.

Qualitatively there is a good agreement between measurements and model results. However there are larger deviations of about $5-10 \%$, especially the exact shape of the forward scattering peak and the wavelength dependence of the degree of polarization are not well reproduced by the model. These deviations can be understood for several possible reasons: First we use aerosol optical properties that we calculated based on the assumption of spherical aerosol particles as model input. Second, OPAC does not include all possible realistic aerosol types. Third, the wavelength dependence of the refractive index in OPAC is probably not exactly the same as for the aerosol measured here. The simulations show that there is a high sensitivity on the aerosol type and that measurements combined with simulations shown here can be used to derive more realistic aerosol properties than those provided by OPAC.

\section{Conclusions}

The Monte Carlo radiative transfer model MYSTIC has been extended by a new efficient and flexible module to simulate polarized solar radiances. The vectorized code has been fully
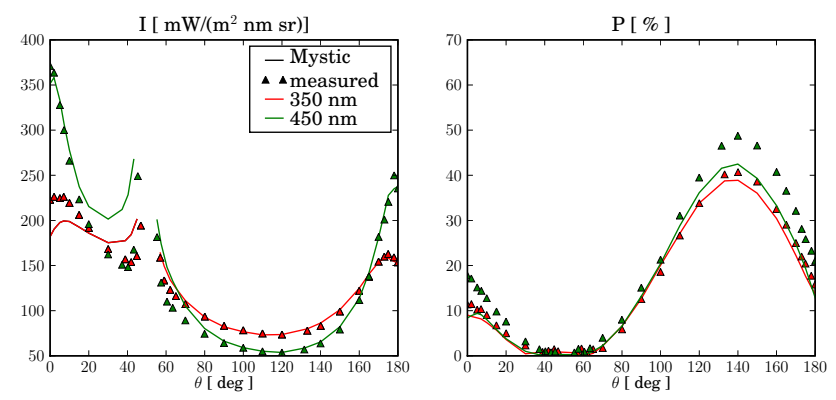

Fig. 13. Intensity and degree of polarization simulated for an aerosol mixture of mineral and water soluble particles (12 June 2005, 10:00 UTC). The aerosol optical thickness was 0.06. Clouds below the measurement site are taken into account using an effective surface albedo of 0.5 .

implemented so that MYSTIC can now consider polarization in horizontally inhomogeneous or spherical atmospheres.

The code has been applied to simulate the polarized sky radiance for pure Rayleigh scattering, for different aerosol types, and for a liquid cloud layer. The polarization pattern of various aerosol types can be very different. Clouds that consist of larger particles also yield a different polarization pattern. Nevertheless, in the Earth's atmosphere the Rayleigh scattering pattern dominates the polarized sky radiance.

The error that is caused by the use of the Delta-M method to approximate the scattering phase matrix has been investigated. As expected the error can be arbitrarily large (a factor of 7 in our example) in the forward scattering region. For an aerosol optical thickness of 0.5 the error in the degree of polarization is about $5 \%$, independent of the direction.

The comparison between ground based radiance measurements and model simulations shows a qualitatively good agreement. However, the exact shape of the forward scattering peak in the radiance measured in the principal plane can not be reproduced. The reason is that the aerosol phase matrices based on the OPAC climatology and on Mie theory, i.e. assuming spherical particles, are not sufficiently realistic. The sensitivity of the forward scattering region and the polarization pattern on the aerosol type shows that multidirectional polarized radiance measurements have a high potential to retrieve information about the aerosol type with unprecedented accuracy.

For reducing complexity and since the horizontal distribution of the aerosols is unknown all simulations were performed in a 1-D spherically symmetric model atmosphere. As mentioned above the code can also be run for a 3D model atmosphere including clouds. Studies about 3-D effects on polarized radiances measured from air-plane (e.g. by the Research Scanning Polarimeter) or from space (e.g. by APS on Glory) are possible model applications that will be exploited in the near future. 
Acknowledgements. We thank C. Kiemle for helpful comments and discussions. Furthermore we thank B. Cairns and the anonymous reviewer for their valuable comments which helped to improve the manuscript significantly. This study was performed within the ESA project ESASLight, ESTEC contract AO/1-5433/07/NL/HE, and the European Commission integrated project SCOUT-O3, contract number 505390-GOCE-CT-2004.

Edited by: J. Quaas

\section{References}

Blanco-Muriel, M., Alarcón-Padilla, D. C., López-Moratella, T., and Lara-Coira, M.: Computing the solar vector, Sol. Energy, 70, 431-441, 2001.

Blumthaler, M., Schallhart, B., Schwarzmann, M., McHenzie, R., Johnston, P., Kotkamp, M., and Shiona, H.: Spectral UV Measurements of Global Irradiance, Solar Radiance, and Actinic Flux in New Zealand: Intercomparison between Instruments and Model Calculations, J. Atmos. Ocean Technol., 25, 945-958, 2008.

Boesche, E., Stammes, P., Ruhtz, T., Preusker, R., and Fischer, J.: Effect of aerosol microphysics on polarization of skylight: sensitivity study and measurements, Appl. Opt., 45, 8790-8805, 2006.

Cahalan, R., Oreopoulos, L., Marshak, A., Evans, K., Davis, A., Pincus, R., Yetzer, K., Mayer, B., Davies, R., Ackerman, T., H.W., B., Clothiaux, E., Ellingson, R., Garay, M., Kassianov, E., Kinne, S., Macke, A., O'Hirok, W., Partain, P., Prigarin, S., Rublev, A., Stephens, G., Szczap, F., Takara, E., Varnai, T., Wen, G., and Zhuraleva, T.: The International Intercomparison of 3D Radiation Codes (I3RC): Bringing together the most advanced radiative transfer tools for cloudy atmospheres, Bulletin of the American Meteorological Society, 86, 1275-1293, 2005.

Cairns, B., Russell, E. E., and Travis, L. D.: Research Scanning Polarimeter: calibration and ground-based measurements, in: Proceedings of SPIE - The International Society for Optical Engineering, 3754, 186-196, 1999.

Cairns, B., Russell, E., LaVeigne, J., and Tennant, P.: Research scanning polarimeter and airborne usage for remote sensing of aerosols, in: Proceedings of SPIE - The International Society for Optical Engineering, 5158, 33-44, 2003.

Chandrasekhar, S.: Radiative transfer, Oxford Univ. Press, UK, 1950.

Chowdhary, J., Cairns, B., Mishchenko, M., and Travis, L.: Constraining aerosol single scattering albedos from multiangle, multispectral photo-polarimetric observations over the ocean, in: Proceedings of SPIE - The International Society for Optical Engineering, 5571, 127-139, 2004.

Collins, D. G., Blättner, W. G., Wells, M. B., and Horak, H. G.: Backward Monte Carlo calculations of the polarization characteristics of the radiation emerging from spherical-shell atmospheres., Appl. Opt., 11, 2684-2696, 1972.

Cornet, C., C-Labonnote, L., and Szczap, F.: Three-dimensional polarized Monte Carlo atmospheric radiative transfer model (3DMCPOL): 3-D effects on polarized visible reflectances of a cirrus cloud, J. Quant. Spectrosc. Radiat. Transfer, 111, 174-186, 2010.

Coulson, K. L., Dave, J. V., and Sekera, Z.: Tables Related to Radiation Emerging from a Planetary Atmosphere with Rayleigh Scattering, University of California Press, 1960.
Davis, C., Emde, C., and Harwood, R.: A 3-D polarized reversed Monte Carlo radiative transfer model for millimeter and submillimeter passive remote sensing in cloudy atmospheres, IEEE Transactions on Geoscience and Remote Sensing, 43, 10961101, 2005.

de Haan, J. F., Bosma, P. B., and Hovenier, J. W.: The adding method for multiple scattering calculations of polarized light, Astron. Astr., 183, 371-391, 1987.

de Rooij, W. A. and van der Stap, C. C. A. H.: Expansion of Mie scattering matrices in generalized spherical functions, Astron. Astr., 131, 237-248, 1984.

Deschamps, P.-Y., Breon, F.-M., Leroy, M., Podaire, A., Bricaud, A., Buriez, J.-C., and Seze, G.: The POLDER mission: instrument characteristics and scientific objectives, IEEE Transactions on Geoscience and Remote Sensing, 32, 598-615, doi: 10.1109/36.297978, 1994.

Emde, C. and Mayer, B.: Simulation of solar radiation during a total eclipse: a challenge for radiative transfer, Atmos. Chem. Phys., 7, 2259-2270, 2007, http://www.atmos-chem-phys.net/7/2259/2007/.

Emde, C., Buehler, S. A., Davis, C., Eriksson, P., Sreerekha, T. R., and Teichmann, C.: A polarized discrete ordinate scattering model for simulations of limb and nadir long-wave measurements in 1-D/3-D spherical atmospheres, J. Geophys. Res. Atmos., 109, 1-20, 2004.

Evans, K. F. and Stephens, G. L.: A new polarized atmospheric radiative transfer model, J. Quant. Spectrosc. Radiat. Transfer, 46, 413-423, 1991.

Hansen, J. E. and Travis, L. D.: Light scattering in planetary atmospheres, Space Sci. Rev., 16, 527-610, 1974.

Hess, M., Koepke, P., and Schult, I.: Optical Properties of Aerosols and Clouds: The Software Package OPAC, B. Am. Meteor. Soc., 79, 831-844, 1998.

IPCC: Climate Change 2007, Tech. rep., Intergovernmental Panel of Global Climate Change, 2007.

Kreuter, A., Zangerl, M., Schwarzmann, M., and Blumthaler, M.: All-sky imaging: a simple, versatile system for atmospheric research, Appl. Opt., 48, 1091-1097, 2009.

Kuik, F., de Haan, J. F., and Hovenier, J. W.: Benchmark results for single scattering by spheroids, J. Quant. Spectrosc. Radiat. Transfer, 47, 477-489, doi:10.1016/0022-4073(92) 90107-F, 1992.

Li, Z., Goloub, P., Devaux, C., Gu, X., Deuzé, J.-L., Qiao, Y., and Zhao, F.: Retrieval of aerosol optical and physical properties from ground-based spectral, multi-angular, and polarized sunphotometer measurements, Remote Sens. Environ., 101, 519533, 2006.

Li, Z., Goloub, P., Dubovik, O., Blarel, L., Zhang, W. andvin, T., Sinyuk, A., Sorokin, M., Chen, H., Holben, B., Tanré, D., Canini, M., and Buis, J.-P.: Improvements for ground-based remote sensing of atmospheric aerosol properties by additional polarimetric measurements, Journal of Quantitative Spectroscopy and Radiative Transfer, 110, 1954-1961, 2009.

Liu, Y. and Voss, K.: Polarized radiance distribution measurement of skylight, II, Experiment and data, Appl. Opt., 36, 8753-8764, 1997.

Marchuk, G. I., Mikhailov, G. A., and Nazaraliev, M. A.: The Monte Carlo methods in atmospheric optics, Springer Series in Optical Sciences, Berlin: Springer, 1980. 
Marshak, A. and Davis, A.: 3-D Radiative Transfer in Cloudy Atmospheres, Springer, ISBN-13 978-3-540-23958-1, 2005.

Mayer, B.: Radiative transfer in the cloudy atmosphere, European Physical Journal Conferences, 1, 75-99, 2009.

Mayer, B. and Kylling, A.: Technical note: The libRadtran software package for radiative transfer calculations - description and examples of use, Atmos. Chem. Phys., 5, 1855-1877, 2005, http://www.atmos-chem-phys.net/5/1855/2005/.

Mie, G.: Beiträge zur Optik trüber Medien, speziell kolloidaler Metallösungen, Annalen der Physik, 330, 377-445, doi:10.1002/ andp.19083300302, 1908.

Mishchenko, M. I., Travis, L., and Lacis, A.: Scattering, Absorption, and Emission of Light by Small Particles, Cambridge University Press, 2002.

Mishchenko, M. I., Cairns, B., Kopp, G., Schueler, C. F., Fafaul, B. A., Hansen, J. E., Hooker, R. J., Itchkawich, T., Maring, H. B., and Travis, L. D.: Accurate Monitoring of Terrestrial Aerosols and Total Solar Irradiance: Introducing the Glory Mission, Bulletin of the American Meteorological Society, 88, 5, doi: 10.1175/BAMS-88-5-677, 2007.

Schulz, F. M., Stamnes, K., and Weng, F.: VDISORT: An improved and generalized discrete ordinate method for polarized (vector) radiative transfer, J. Quant. Spectrosc. Radiat. Transfer, 61, 105$122,1999$.

Stamnes, K., Tsay, S.-C., Wiscombe, W., and Jayaweera, K.: Numerically stable algorithm for discrete-ordinate-method radiative transfer in multiple scattering and emitting layered media, Appl. Opt., 27, 2502-2509, 1988. van de Hulst, H. C.: Light Scattering by Small Particles, Dover, 1981.

Vermeulen, A., Devaux, C., and Herman, M.: Retrieval of the scattering and microphysical properties of aerosols from groundbased optical measurements including polarization, I, Method, Appl. Opt., 39, 6207-6220, 2000.

Warren, S. G.: Optical constants of ice from the ultraviolett to the microwave, Appl. Opt., 23, 1206-1225, 1984.

Wauben, W. M. F. and Hovenier, J. W.: Polarized radiation of an atmosphere containing randomly-oriented spheroids, J. Quant. Spectrosc. Radiat. Transfer, 47, 491-504, doi:10.1016/ 0022-4073(92)90108-G, 1992.

Weihs, P., Lenoble, J., Blumthaler, M., Martin, T., Seckmeyer, G., Philipona, R., la Casiniere, A. D., Sergent, C., Gröbner, J., Cabot, T., Masserot, D., Pichler, T., Pougatch, E., Rengarajan, G., Schmucki, D., and Simic, S.: Modeling the effect of an inhomogeneous surface albedo on incident UV radiation in mountainous terrain: determination of an effective surface albedo, Geophys. Res. Lett., 28, 3111-3114, 2001.

Wiscombe, W.: Improved Mie scattering algorithms, Appl. Opt., 19, 1505-1509, 1980.

Wiscombe, W. J.: The Delta- $M$ method: rapid yet accurate radiative flux calculations for strongly asymmetric phase functions, J. Atmos. Sci., 34, 1408-1422, 1977. 